\title{
A Novel Design of Electro-Hydraulic Driven Active Powered Ankle-Foot Prosthesis
}

Qitao Huang ( $\sim$ huangqitao@hit.edu.cn )

Harbin Institute of Technology https://orcid.org/0000-0002-3065-5820

Bowen Li

Harbin Institute of Technology

Fei Jia

Harbin Institute of Technology

\section{Original Article}

Keywords: Ankle-foot prosthesis, EHA, Simulation

Posted Date: February 4th, 2021

DOl: https://doi.org/10.21203/rs.3.rs-168142/v1

License: (1) This work is licensed under a Creative Commons Attribution 4.0 International License.

Read Full License 


\section{Title page}

\section{A Novel Design of Electro-hydraulic Driven Active Powered Ankle-Foot Prosthesis}

Qi-Tao Huang, born in 1977, is currently an associate professor at Department of Fluid Control and Automation, Harbin Institute of Technology, China. His main research interests include mechachonics engineering, fluid power transmission and control.

E-mail: huangqitao@hit.edu.cn

Bo-Wen Li, born in 1992, is currently a master candidate at Department of Fluid Control and Automation, Harbin Institute of Technology, China. He received his bachelor degree from Northeastern University, China, in 2015. His research interests include biomechanics and intelligent robotics.

E-mail: 19s108212@stu.hit.edu.cn

Jia-Fei, born in 1999, is currently a master candidate at Department of Fluid Control and Automation, Harbin Institute of Technology, China. He received his bachelor degree from Northeast Agricaulture University, China, in 2020. His research interests include fluid control, biomechanics and intelligent robotics.

E-mail: 19s108212@stu.hit.edu.cn

Corresponding author: Qi-Tao Huang E-mail: huangqitao@hit.edu.cn 


\title{
A Novel Design of Electro-hydraulic Driven Active Powered Ankle-Foot Prosthesis
}

\author{
Qi-Tao Huang $•$ Bo-Wen Li $\cdot$ Jia-Fei
}

Harbin Institute of Technology, China.

Received xx xx, 2021; revised xx xx, 2021; accepted xx xx, 2021

\begin{abstract}
This paper presents the design and control of a novel full active powered ankle prosthesis driven by electrohydrostatic actuator (EHA) to improve amputee gait during the level-ground walking in full-time gait cycle. A $100 \mathrm{~W}$ brushless DC motor driving a $0.92 \mathrm{cc} / \mathrm{rev}$ bi-directional gear pump serves as the power kernel. An elastic element is configured in series with the hydraulic cylinder. With this architecture, better power characteristic, ability of energy storage and passive compliance are obtained to revive sound human characteristic as much as possible. To smooth the gait pattern, a neuromuscular model with Hill-type muscle tendon structure is introduced into the control system scheduled by finite state machine which was designed to carry on different control strategy during individual gait phase.
\end{abstract}

Keywords: Ankle-foot prosthesis $\bullet$ EHA $・$ Simulation

\section{Introduction}

Millions of people are suffering from lower limb amputations worldwide, including 1.6million in China alone, while the number is continuously growing due to illness and accidental injury. Wearing prosthesis is the most effective way to restore basic daily activities under the current medical level. Conventional passive prosthesis is designed utilizing a series of composite materials which can storage and release energy during locomotion[1]. This kind of prosthesis duplicate the function of human tendon having advantages of small size and light weight, but obviously, has shortcoming of lacking the ability of provide motivation. This results in amputees with passive ankle-foot prosthesis having lower walking speed, more energy consumption and distinctly asymmetric gait pattern especially for unilateral transtibial amputees[2].

To overcome the defects of the passive prosthesis, powered prosthesis, putting forward by researchers a few decades ago, is enjoying rapid development in recent years which have the able to duplicate the kinematic and kinetic characteristics of the human body one step further [3].

When it comes to active or rather powered domain, power

Qi-Tao Huang

huangqitao@hit.edu.cn

1Department of Fluid Control and Automation, Harbin Institute of to mass/volume ratio turns into the maximal limitation. According to research[4], the below-knee complex of a medium weight $(75 \mathrm{~kg})$ person weighs approximately $2 \mathrm{~kg}$ which meanwhile can produce up to $350 \mathrm{~W}$ and $140 \mathrm{Nm}$ peak power and torque. Under current research level, it is arduous to match both weight and drive capability, thus powered ankle prostheses are significantly less commercial. A variety of kernels has been attempted by researchers to provide net power output, including pneumatic actuation[5], pneumatic artificial muscles [6] and series-elastic actuator (SEA)[7]. Electro-hydrostatic actuator is a power-by-wire (PBW) servo system widely used in aerospace industry which is appropriate for a powered ankle prosthesis due to its high output power to mass ratio, good controllability and robustness[8]. Our team had developed an ankle-foot prosthesis, the control strategy of which is active-passive hybrid to provide both positive force output during powered plantar flexion and damping in other passive phase to relieve shock load with introducing directional control valve and throttle valve in the circuit. However, the hydraulic system of the design is much complex and the mechanical structure of which is lack of energy storage device. Due to having those defects, it cannot provide adequate force output according to our previous experiment and the damping is not sufficient either due to the internal leakage of the hydraulic cylinder and pump. In addition, the stiffness of the prosthesis is not able to be conducted during the stance phase which play a leading role in the dynamics of walking process.

To provide sufficient power output and duplicate the dynamic characteristics of sound body, this paper proposes a novel EHA based full-time active prosthesis. The hydraulic system is streamlined and an elastic element is introduced into to form the series elastic actuator (SEA) to change the energy output pattern. Furthermore, Neuromuscular model is introduced into the control system serving as a feedforward element to duplicate the nonlinear impedance and active power output characteristics of human ankle joint.

Technology , Harbin, 150001, China 


\section{Ankle foot Prosthesis}

The EHA powered ankle prosthesis used in this study is an improved successor to the throttle-based MK-I tethered prototype developed by our group. As shown in Figure 1(a), the sole is designed referring to the Flex-Foot[9], a passive carbon fiber made structure absorbing the ground contact shock to the amputee while storing part of the energy like traditional ankle-foot prostheses do. The ankle joint is a simple rolling bearing-based unit joining the sole to upper shank structure then providing a limited range of fixed-axis rotation.

To get rid of the throttling valves to avoid unnecessary high pressure loss, a brushless DC motor (Maxon ECi-40 operating at $48 \mathrm{~V}$ ) and a bi-directional gear pump (VIVOLO $\mathrm{XV}-0 \mathrm{R} / 0.98$ ) were used to deliver hydraulic oil directly to either side of the ankle cylinder via a very short pipeline integrated inside the manifold block. According to simulation result, the pressure loss was signally reduced. The hydraulic circuit is shown in Figure 1(b), a pneumatic source supplies the oil tank working as an accumulator to charge the low-pressure side through the check valve of the loop to avoid cavitation. The prosthesis system will operate in fullactive mode during the whole gait cycle and a relatively complex low-level controller was designed to drive (or backdrive against the flow) the motor-pump unit in stance phase generating desired impedance which covers both damping and stiffness.

A National Instruments (NI) CompactRIO was selected as the control platform handling signal acquisition, overall control and communications for the ankle-foot prosthesis. The motor is controlled by an Escon 50/5 servo controller which is able to record the motor speed in the meantime. Design specifications and parameters of components can be seen in our previous work[10].

There is a series spring configured in series with the cylinder. We combine the EHA system and the series spring to form the Series-Elastic Actuator (SEA). The combination of actuators and springs is a biological substitute for muscle and Achilles tendon respectively. The SEA provides force control by controlling the extent to which the series spring is compressed. Using pressure sensors configured on each port of hydraulic cylinder, we get the force output by multiply the pressure difference and the cylinder area. The design of the spring parameters will be developed in the following chapter. (a)

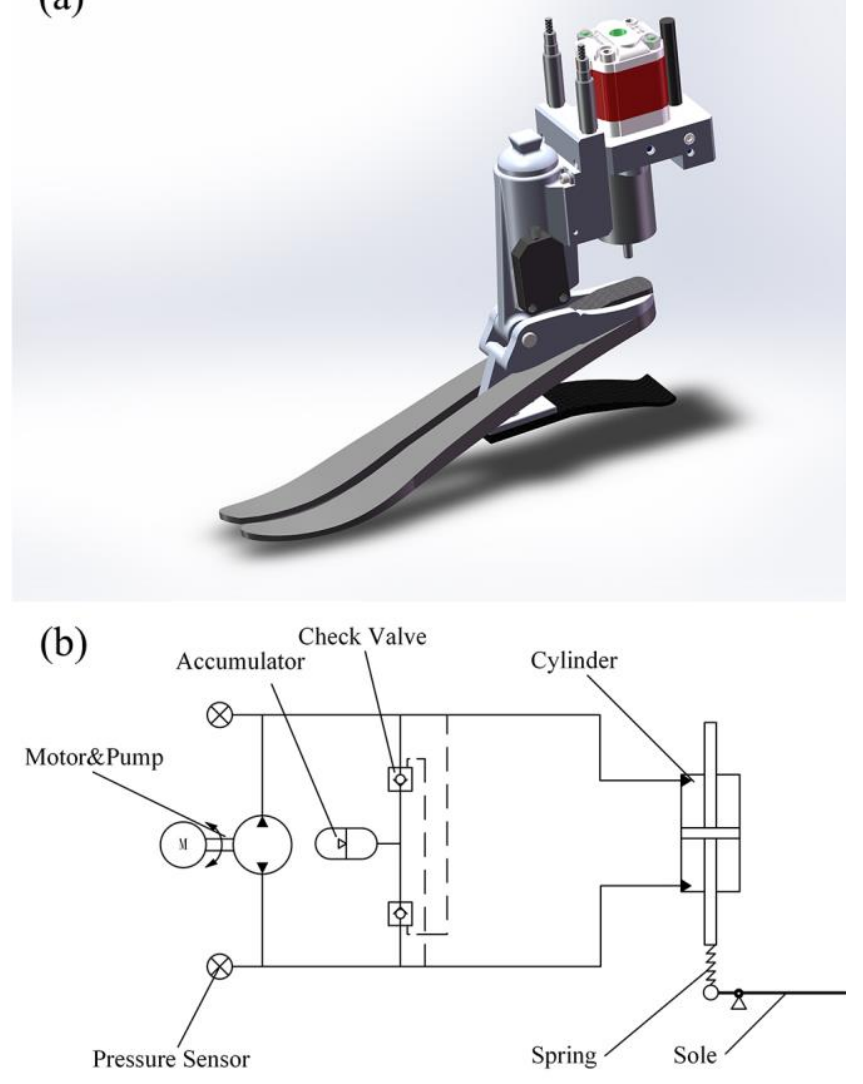

Figure 1 (a) CAD mechanical structure, (b) hydraulic schematic diagram.

\section{Series Spring}

According to the biomechanics, the dynamics of the ankle are mainly nonlinear elastic characteristics during the controlled dorsiflexion which has the proportion of time in stance phase[11]. During the controlled dorsiflexion(CD) phase, some of the gravitational potential energy is stored in the Achilles tendon and released during the powered plantarflexion(PP) phase, reducing the burden on the muscle's force output. Obviously, with the same joint torque, lower stiffness allows the spring to store more energy. Nevertheless, too low stiffness will result in insufficient force bandwidth, a widely accepted measure of an actuator's ability to achieve a desired performance[12], thus cannot meet the needs of amputees to walk at a specific speed. Research[13]had pointed out that, the goal of the force bandwidth is $2-4 \mathrm{~Hz}$ at $50-140 \mathrm{Nm}$ torque during human level-ground walking.

Table 1 Main parameters for calculating the force bandwidth

\begin{tabular}{cll}
\hline Symbol & Specification & Value \\
\hline$\omega_{0}$ & Motor maximum speed & $5000 \mathrm{rpm}$ \\
$D$ & Pump displacement & $0.92 \mathrm{~cm}^{3} / \mathrm{rev}$ \\
$C_{i c}$ & Cylinder Internal & $2 \times 10^{-12} \mathrm{~m}^{3} /(\mathrm{Pa} \cdot \mathrm{s})$ \\
& Leakage Coefficient & \\
\hline
\end{tabular}




\begin{tabular}{cll}
\hline$C_{i p}$ & $\begin{array}{l}\text { pump Internal Leakage } \\
\text { Coefficient }\end{array}$ & $1.46 \times 10 \mathrm{~m}^{3} /(\mathrm{Pa} \cdot \mathrm{s})$ \\
$K_{m}$ & $\begin{array}{l}\text { Motor velocity-torque } \\
\text { coefficient }\end{array}$ & $1160 \mathrm{rpm} / \mathrm{Nm}$ \\
$K_{f}$ & $\begin{array}{l}\text { Pump Pressure-based } \\
\text { Friction Coefficient }\end{array}$ & $1.07 \times 10^{-3} \mathrm{Nm} / \mathrm{bar}$ \\
$A$ & $\begin{array}{l}\text { Cylinder Working Area } \\
\text { nat }\end{array}$ & $6.5 \mathrm{~cm}^{2}$ \\
\hline
\end{tabular}

To determine the spring stiffness by force bandwidth requirement, the EHA model is analyzed. Firstly, the motor is not an ideal torque source. The torque output of the motor decreases with the increase of the motor speed due to the influence of the back electromotive force. The motor speed can be described as (1):

$$
\omega(s)=\omega_{0}-K_{m} \cdot T_{f}(s)
$$

where $\omega_{0}$ is the maximum speed of the motor, $K_{m}$ is the velocity-torque coefficient, $T_{f}$ is the load torque.

Because the motor and gear pump are directly connected through the coupling, the gear pump load torque is the motor by the load torque. The load of gear pump is given by Formula (2), while (3) is the force equation of the hydraulic cylinder.

$$
\begin{gathered}
T_{f}(s)=\Delta p(s)\left(D+K_{f}\right) \\
F_{L}(s)=\Delta p(s) \cdot A
\end{gathered}
$$

Where $\Delta p$ is the pressure difference across the pump; $D$ is the pump displacement; $k_{f}$ is pressure-based friction coefficient; $F_{L}$ is the load of hydraulic cylinder; $A$ is the working area of the cylinder.

Because the hydraulic cylinder volume is small and the working pressure is low, the change of the liquid volume due to the pressure is ignored.

$$
D \omega(s)=\left(C_{i c}+C_{i p}\right) \Delta p(s)+A s \cdot x(s)
$$

Where $C_{i c}$ is the cylinder leakage coefficient, $C_{i p}$ is the pump leakage coefficient, $x$ is the displacement of cylinder piston.

According to the force equation of the spring (5), we get the force output of the cylinder under motor saturation (6)

$$
\begin{gathered}
F_{L}=k_{s} \cdot x(s) \\
F_{L}=\frac{k_{s} A \omega_{0} D}{A^{2} s+k_{s}\left(k_{m} D\left(D+k_{f}\right)+C_{i c}+C_{i p}\right)}
\end{gathered}
$$

In the same way, we can get the maximum output (7) of the hydraulic cylinder in the saturation of the motor, with considering the leakage and friction of hydraulic element. Finally, we have the normalized bandwidth equation (8).

$$
\begin{gathered}
F_{s a t}=\frac{A \omega_{0} D}{k_{m} D\left(D+k_{f}\right)+C_{i c}+C_{i p}} \\
\frac{F_{l}}{F_{s a t}}=\frac{1}{s \frac{A^{2}}{k_{s}\left(k_{m} D\left(D+k_{f}\right)+C_{i c}+C_{i p}\right)}+1}
\end{gathered}
$$

Through the above analysis and calculation, we had got that the stiffness of $2000 \mathrm{KN} / \mathrm{m}$ is a relatively appropriate stiffness value. The result is shown in the Figure 2, the bandwidth of which is about $9.9 \mathrm{HZ}$ at $110 \mathrm{Nm}$ torque output.

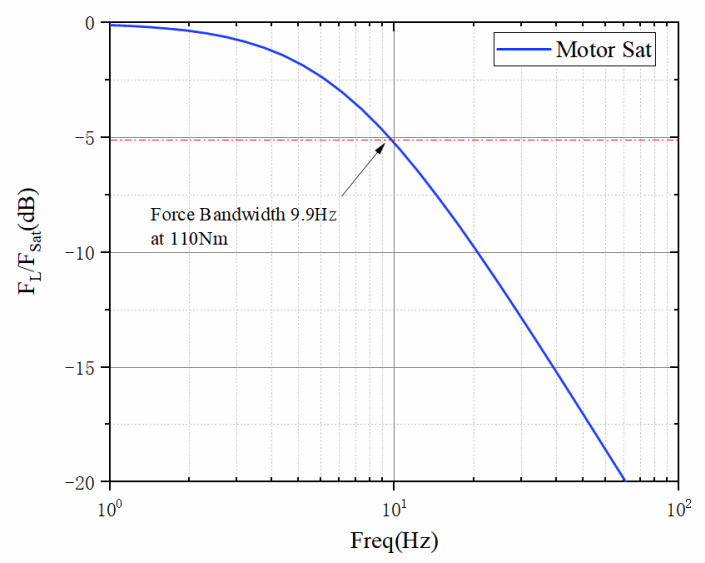

Figure 2 The force bandwidth of when maximum output force drops below the peak force required.

To save space and not attach too much extra weight and guarantee the power density of the prosthetic system, this paper selects the cantilever structure to design a spring as shown in Figure 3. The design adopts the cantilever beam with rectangular cross section, the stiffness of which is determined by the deflection formula (9).

$$
K_{s p}=\frac{3 E I}{l_{s}^{3}}=\frac{E h^{2} w_{s}}{4 l_{s}^{3}}
$$

\section{Simulation model}

Before clinical evaluation, a simulation experiment is carried out in Matlab/Simulink to verify its feasibility. The spring part was not added in the previous model of prosthetic ankle[10], while it was added in the simulation of this paper. Meanwhile, pressure loss is ignored, for the hydraulic circuit 


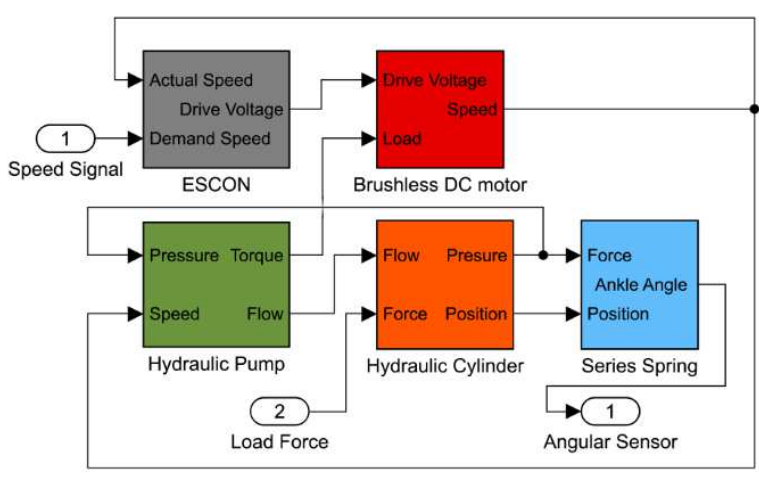

Figure 3 The Simulink model of the ankle-foot prosthesis.

are greatly simplified compared with the those in MK-I. The simulation block diagram is shown in Figure 4.

What we desire is an arm exoskeleton which is capable of following motions of the human upper-limb accurately and supplying the human upper-limb with proper force feedback if needed. In order to achieve an ideal controlling performance, we have to examine the structure of the human upper-limb.

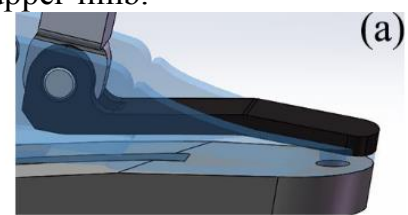

(a)

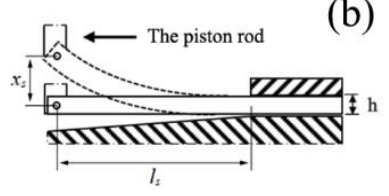

Figure 4 (a)The CAD structure of the spring, (b) Mechanical schematic diagram of the cantilever beam.

\section{Control System}

The design concept of the control system is restoring the dynamic characteristics of a sound ankle in level ground walking and small gradient adaption, in a word, constructing a real-time relationship between ankle angle measured and ankle torque produced by the prosthesis. As aforementioned, the controller designed is able to determine the corresponding torque using feedforward with neuromuscular model as kernel, in which, a single degree of freedom (DOF) hinge is used to represent human ankle joint. A Hill type muscle-tendon structure (MTS) is attached between the heel and upper shank in the model like the soleus and Achilles tendon in an intact human lower limb. During early stance and swing phase, the MTS will stay slack and won't produce negative force, therefore, a spring-damper based dorsiflexor rather than a second MTS is introduced for simplicity. A finite-state machine (FSM) is used as high-level controller to estimate the gait phase of the amputee on the basis of sensor signals. These two virtual actuators work in alterable mode in each individual gait phase and the output torque command of the feedforward model is a combination of their respective torque contribution. Since the EHA is force controllable[14], the physical torque at the ankle joint produced by the prosthesis will follow the command torque. Neuromuscular model is a Hill-type MTS with positive force reflex scheme, which is of the most importance in the control system since it is the base of the non-linear impedance characteristics. The MTS consists of two major parts: an active element called contractile element (CE) models the muscular tissue and a series element (SE) models the tendon. More specifically, the CE consists of three components: A Hill-type muscle and a high-limit parallel elasticities models the connective tissue that surrounds the $\mathrm{CE}[15]$. The unidirectional force generated by the $\mathrm{CE}$ is a function of the $\mathrm{CE}$ length $l_{C E}$, velocity $v_{C E}$ and active state $\mathrm{A}[16]$.

The resulting force $F_{C E}$, referring[17], can be described by

$$
F_{C E}\left(l_{C E}, v_{C E}, A\right)=F_{\max } f_{L}\left(l_{C E}\right) f_{v}\left(v_{C E}\right) A
$$

The SE can be simply characterized by a non-linear elastic force-length relationship (van Ingen Schenau,1984) described by

$$
F_{S E}(\varepsilon)= \begin{cases}F_{\text {max }}\left(\varepsilon / \varepsilon_{r e f}\right)^{2}, & \varepsilon>0 \\ 0, & \varepsilon \leq 0\end{cases}
$$

Where $\varepsilon$ is tendon strain defined by $\varepsilon=\left(l_{S E}-l_{\text {slack }}\right) / l_{\text {slack }}$, lslack is the SE's rest length and $\varepsilon_{\text {ref }}$ is the reference strain obtained by $F_{S E}\left(\varepsilon_{\text {ref }}\right)=F_{\max }$.

Since the CE and SE are in series, ignoring the effect of their negligible mass, they have equal force defined as $F_{M T S}$. Combining the equations aforementioned, the $F_{M T S}$ can be uniquely determined for a specific given MTS length $l_{M T S}(\mathrm{t})$ and real-time active state $A(t)$.

As aforementioned, the synergy as well as the working mode of the two virtual actuators are controlled by a finite-state machine based decisive controller. A gait cycle is divided into three phases based on the variational environment interaction of the prosthesis: early stance (controlled dorsiflexion), late stance and swing. The detection and switch between phases are triggered by the decisive controller as shown in Figure 5.

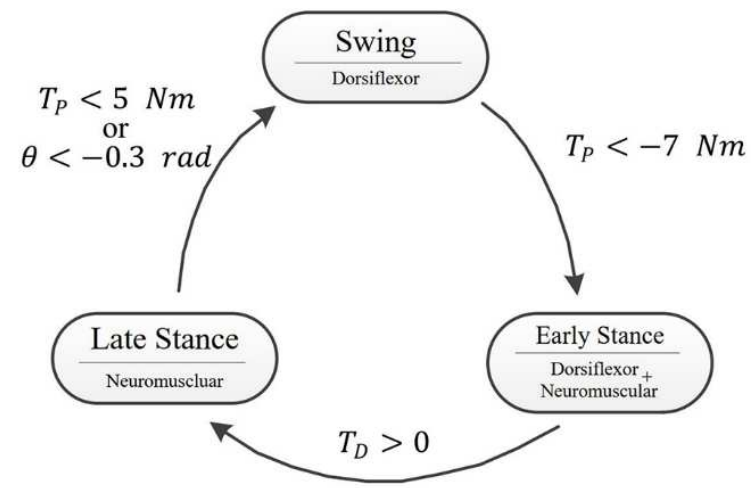

Figure 5 Block diagram of state machine with transition thresholds.

In addition, the torque command is multiplied by a gain coefficient $K_{c}$ to compensate the attenuation in force margin 
of the prosthesis before sent into the force controller. Combining the feedforward model, the compensation gain and the force controller, the architecture of the control system is shown in Figure 6.

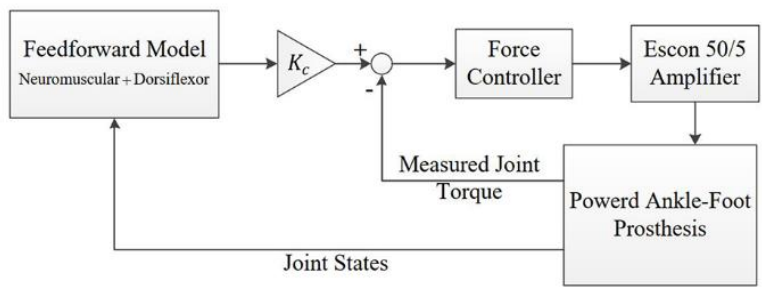

Figure 6 The control system of the powered ankle-foot prosthesis.

\section{Results And Discussion}

The simulation experiment model consists of the two parts mentioned above. The presampled biological ankle torque from[10]is adopted as the input of the ankle prosthesis system model simulating the interaction among the upperlimb, the prosthesis and the ground in form of moment. The simulation result of ankle angle is shown in Figure 7 with matched groups, where dotted line refers to the angular displacement of the actuator, dashed line refers to the angular displacement of the ankle prosthesis and the solid line is the angle profile of sound body. Due to the introduction of the spring, the actual dorsiflexion angle of the actuator is reduced by about 5 degrees, which significantly reduced the velocity of the actuator during the power plantarflexion phase. And this result in a $35 \mathrm{w}$ drop in peak power, as shown in Figure 8 where dotted line refers to the ankle power output without elastic element, full line refers to ones with spring.

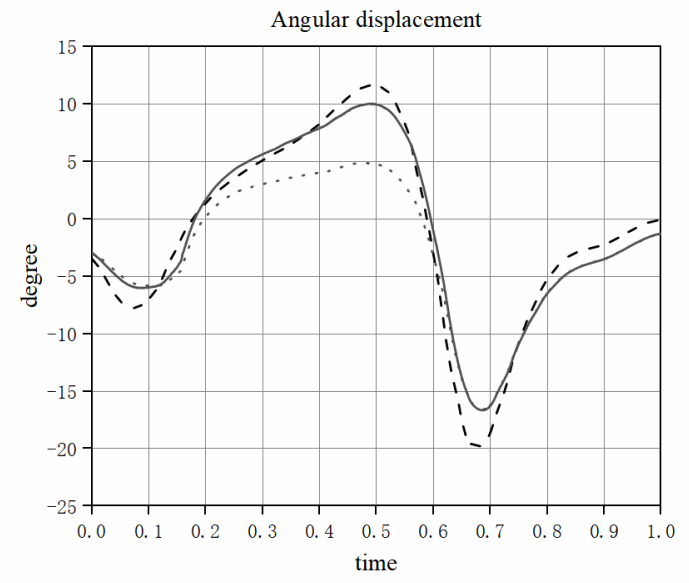

Figure 7 Ankle angle with matched groups of a pre-sampled biological ankle angle profile and the angular displacement of actuator.

\section{Conclusions}

According to the simulation result, the novel design of EHA is able to duplicate the dynamic characteristics of sound human body during level walking. Comparing to the design without elastic element, prosthesis with SEA can change the displacement of actuator, and reduce the required flow rate significantly, which can reduce the peak power produced by the motor. To ensure that the ability of power output isn't affected by the plastic element, evaluation of the force bandwidth is needed. The actual situation may be different from the simulation result, while adjust may need when constructing the prototype.

The future work will continue focus on optimizing the drive system. Hugh Her and his team proposed an optimization method for motor driven lowerlimb prosthesis combining motor selection, transmission ratio optimization, and spring design, so as to improve the performance of the prosthesis system in terms of energy consumption[12].Similarly, our team will explore specific optimization schemes for EHAbased ankle-foot prosthesis.

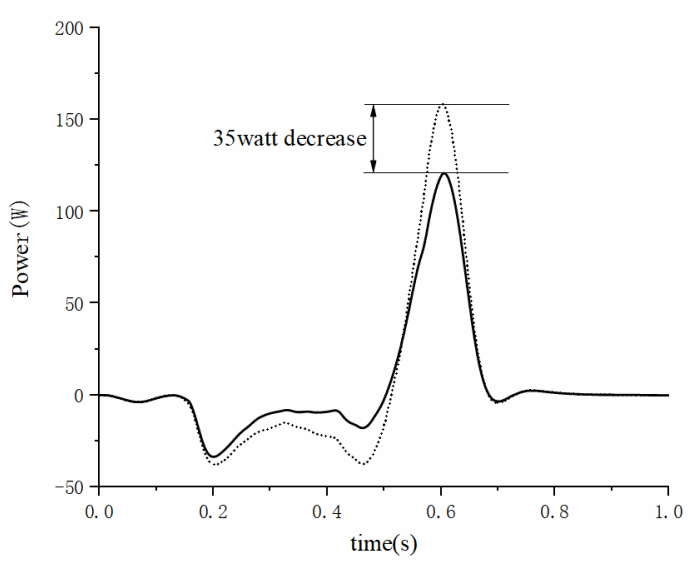

Figure 8 Ankle power output comparison.

\section{Acknowledgements}

\section{Declarations}

\section{Acknowledgements}

The authors would like to thank the support of National Key Research and Development Program of China (No. 2018YFB1307303).

\section{Funding}

This research was supported by National Key R\&D Program of China, Research and application of key technology of intelligent powered lower-limb prosthesis system project (2018YFB1307300).

\section{Availability of data and materials}

The datasets supporting the conclusions of this article are included within the article. 


\section{Authors' contributions}

The author' contributions are as follows: Huang Qitao was in charge of the whole trial and electro-hydraulic system design; Li Bowen assisted with sampling and laboratory analyses and wrote the manuscript; Jia Fei assisted with simulation and laboratory analyses.

\section{Competing interests}

The authors declare no competing financial interests.

\section{Consent for publication}

Not applicable

\section{Ethics approval and consent to participate} Not applicable

\section{References}

[1] Seymour R. Prosthetics and orthotics: lower limb and spinal[M]. Lippincott Williams \& Wilkins, 2002.

[2] Breakey J. Gait of unilateral below-knee amputees[J]. Orthotics and Prosthetics, 1976, 30(3): 17-24.

[3] Jimenez-Fabian R, Verlinden O. Review of control algorithms for robotic ankle systems in lower-limb orthoses, prostheses, and exoskeletons[J]. Medical engineering \& physics, 2012, 34(4): 397-408.

[4] Au S K, Dilworth P, Herr H. An ankle-foot emulation system for the study of human walking biomechanics[C]//Proceedings $2006 \quad$ IEEE International Conference on Robotics and Automation, 2006. ICRA 2006. IEEE, 2006: 2939-2945.

[5] Sup F, Bohara A, Goldfarb M. Design and control of a powered transfemoral prosthesis[J]. The International journal of robotics research, 2008, 27(2): 263-273.

[6] Versluys R, Desomer A, Lenaerts G, et al. A pneumatically powered below-knee prosthesis: Design specifications and first experiments with an amputee[C]//2008 2nd IEEE RAS \& EMBS International Conference on Biomedical Robotics and Biomechatronics. IEEE, 2008: 372-377.

[7] Hitt J K, Sugar T G, Holgate M, et al. An active footankle prosthesis with biomechanical energy regeneration[J]. Journal of medical devices, 2010, 4(1).

[8] Yu T, Plummer A, Iravani P, et al. The design of a powered ankle prosthesis with electrohydrostatic actuation[C]//Fluid Power Systems Technology. American Society of Mechanical Engineers, 2015, 57236: V001T01A041.

[9] Ossur, (2020). Retrieved December 27,2020, from Össur:http://www.ossur.com

[10] Liu H, Huang Q, Tong Z. Simulation and Analysis of a Full-Active Electro-Hydrostatic Powered Ankle Prosthesis[C]//2019 19th International Conference on Advanced Robotics (ICAR). IEEE, 2019: 81-86.

[11] Palmer M L. Sagittal plane characterization of normal human ankle function across a range of walking gait
speeds[D]. Massachusetts Institute of Technology, 2002.

[12] Carney M, Herr H M. Energetic Consequences of Series and Parallel Springs in Lower-Extremity Powered Prostheses[J]. 2019.

[13] Au S K, Weber J, Herr H. Biomechanical design of a powered ankle-foot prosthesis[C]//2007 IEEE 10th International conference on rehabilitation robotics. IEEE, 2007: 298-303.

[14] Yongling F U, Xu H A N, Sepehri N, et al. Design and performance analysis of position-based impedance control for an electrohydrostatic actuation system[J]. Chinese Journal of Aeronautics, 2018, 31(3): 584-596.

[15] Winter D A. Biomechanics and motor control of human gait: normal, elderly and pathological[M]. 1991.

[16] van Soest A J, Bobbert M F. The contribution of muscle properties in the control of explosive movements[J]. Biological cybernetics, 1993, 69(3): 195-204.

[17] Geyer H, Seyfarth A, Blickhan R. Positive force feedback in bouncing gaits?[J]. Proceedings of the Royal Society of London. Series B: Biological Sciences, 2003, 270(1529): 2173-2183. 
Figures

(a)
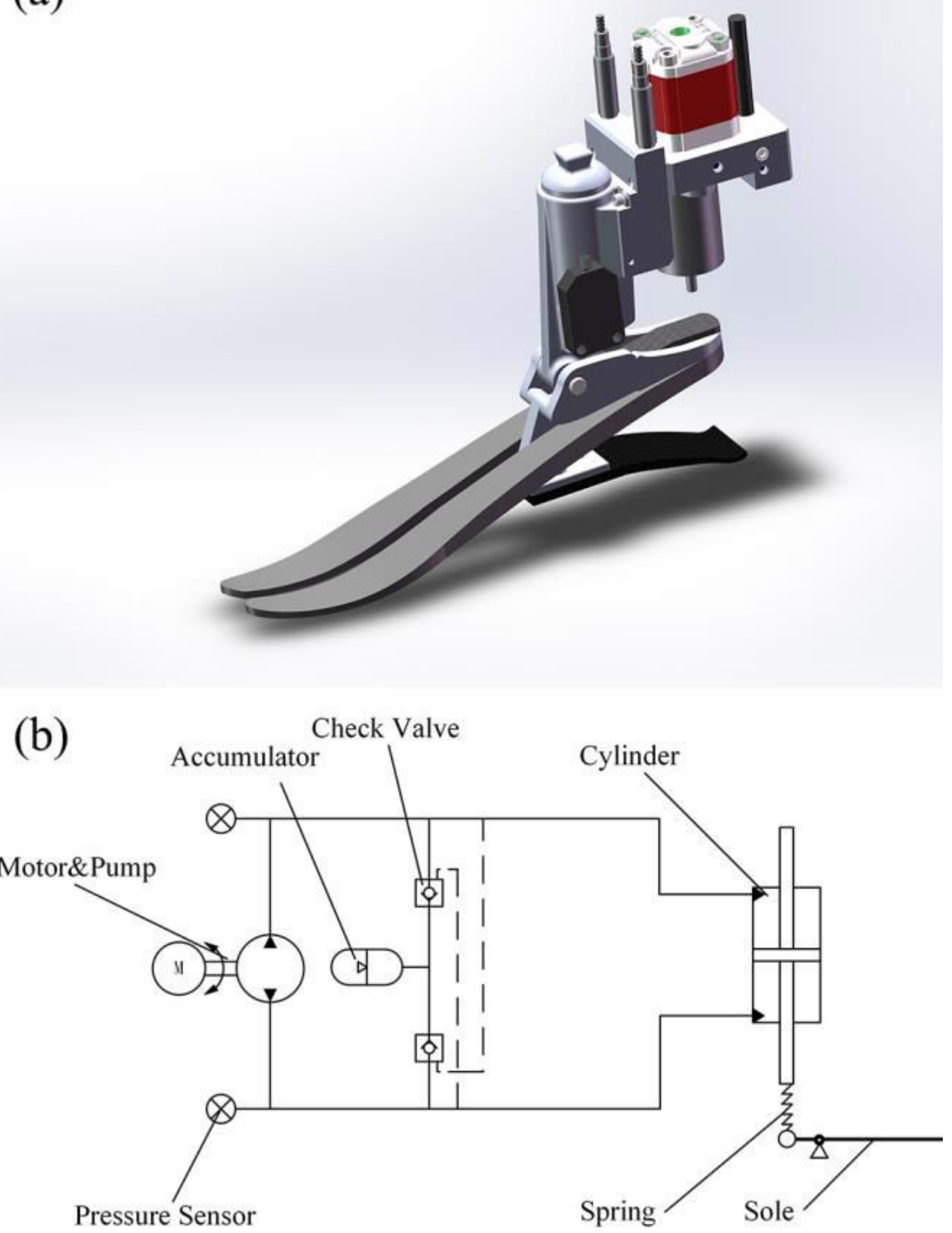

Figure 1

(a) CAD mechanical structure, (b) hydraulic schematic diagram. 


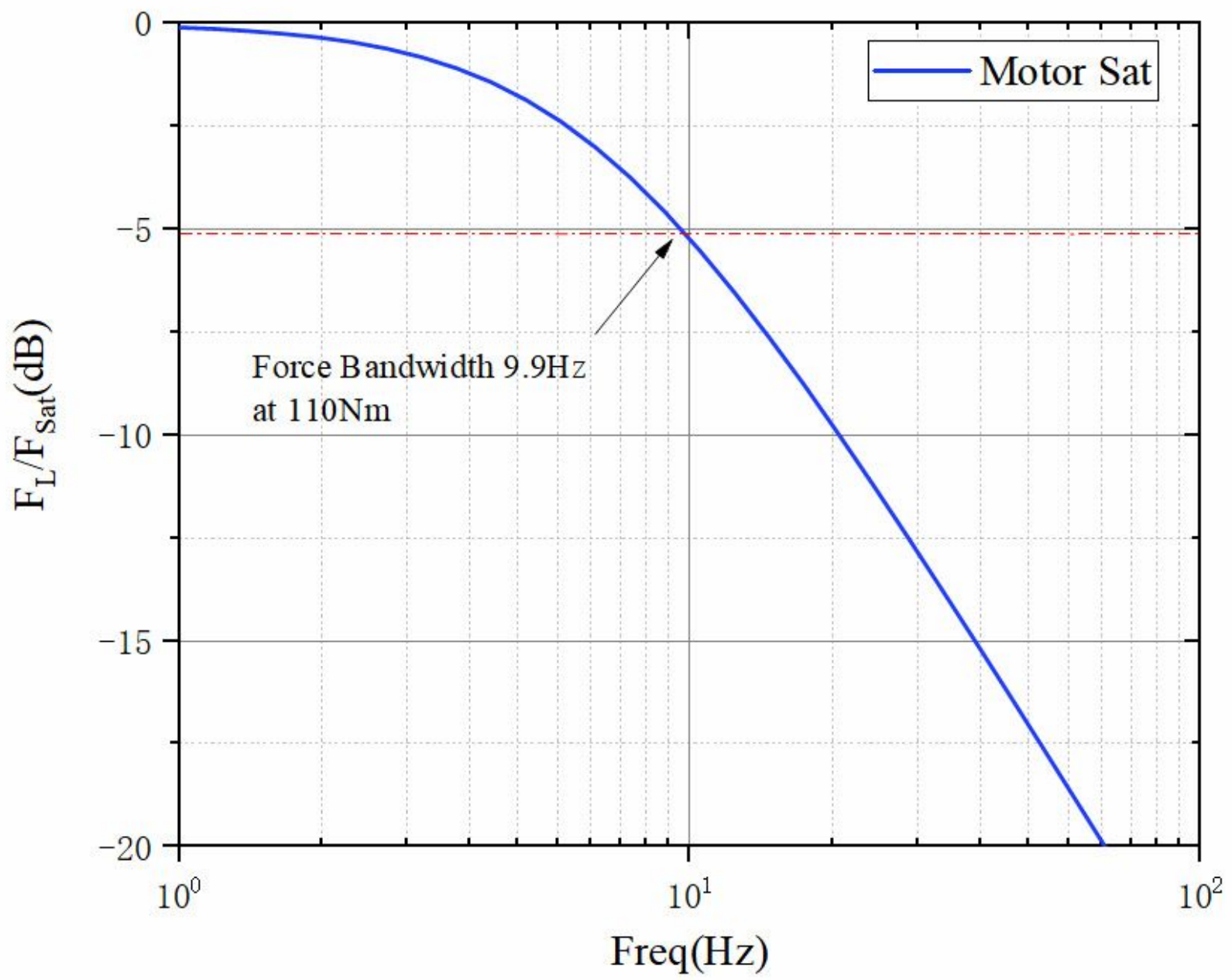

Figure 2

The force bandwidth of when maximum output force drops below the peak force required. 


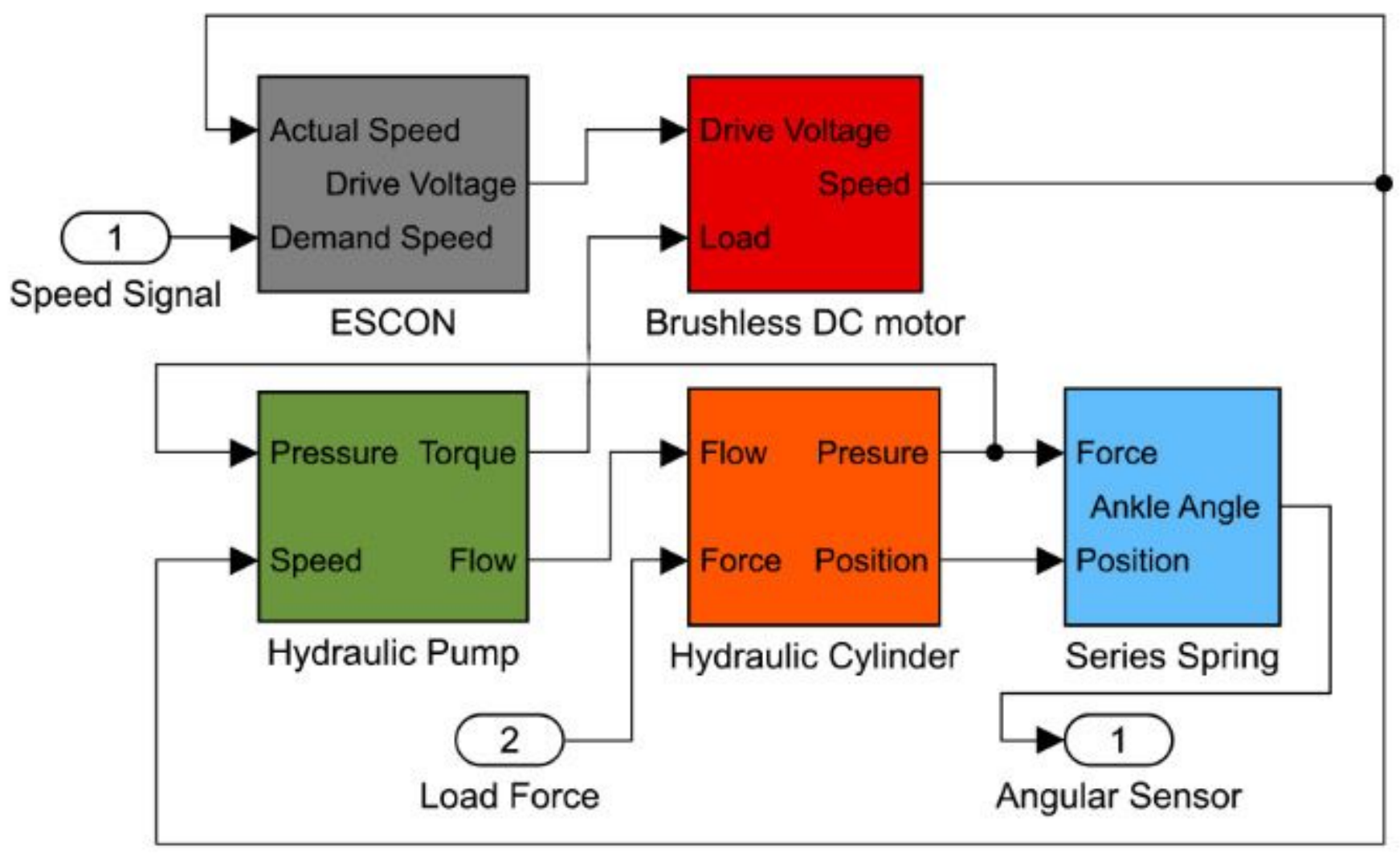

Figure 3

The Simulink model of the ankle-foot prosthesis.
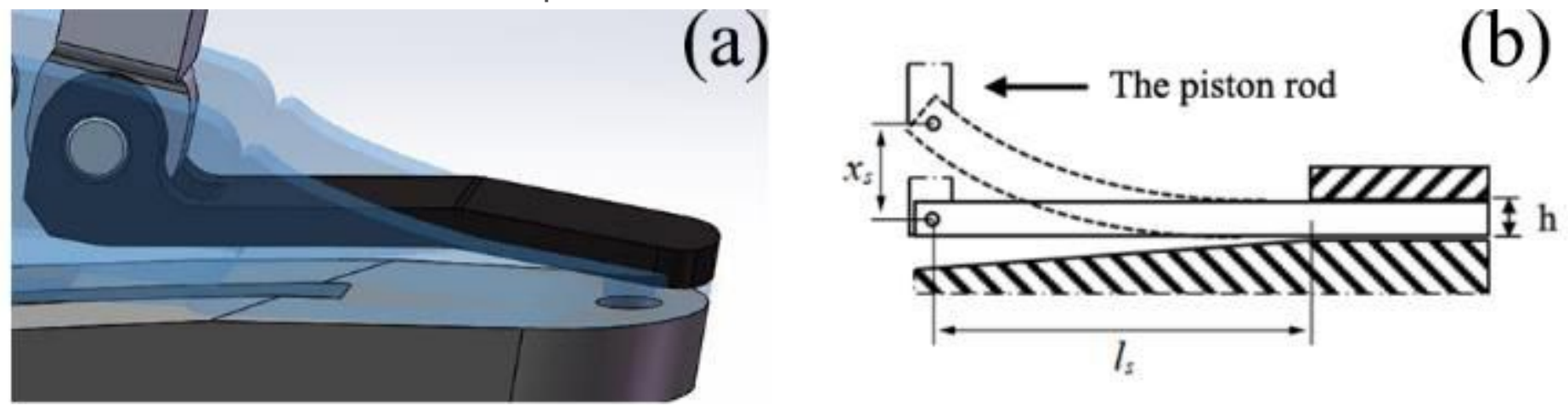

Figure 4

(a)The CAD structure of the spring, (b) Mechanical schematic diagram of the cantilever beam. 


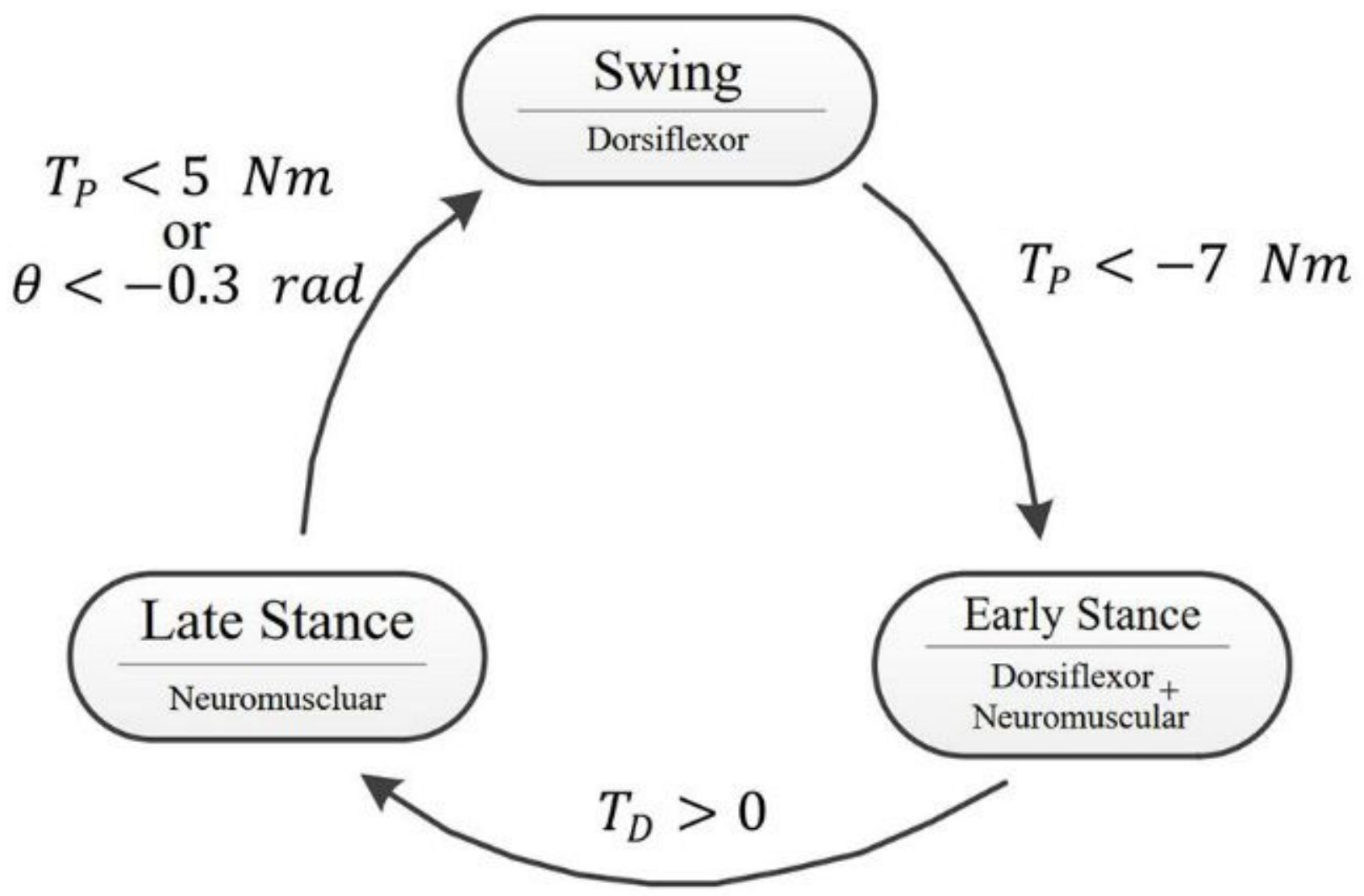

Figure 5

Block diagram of state machine with transition thresholds.

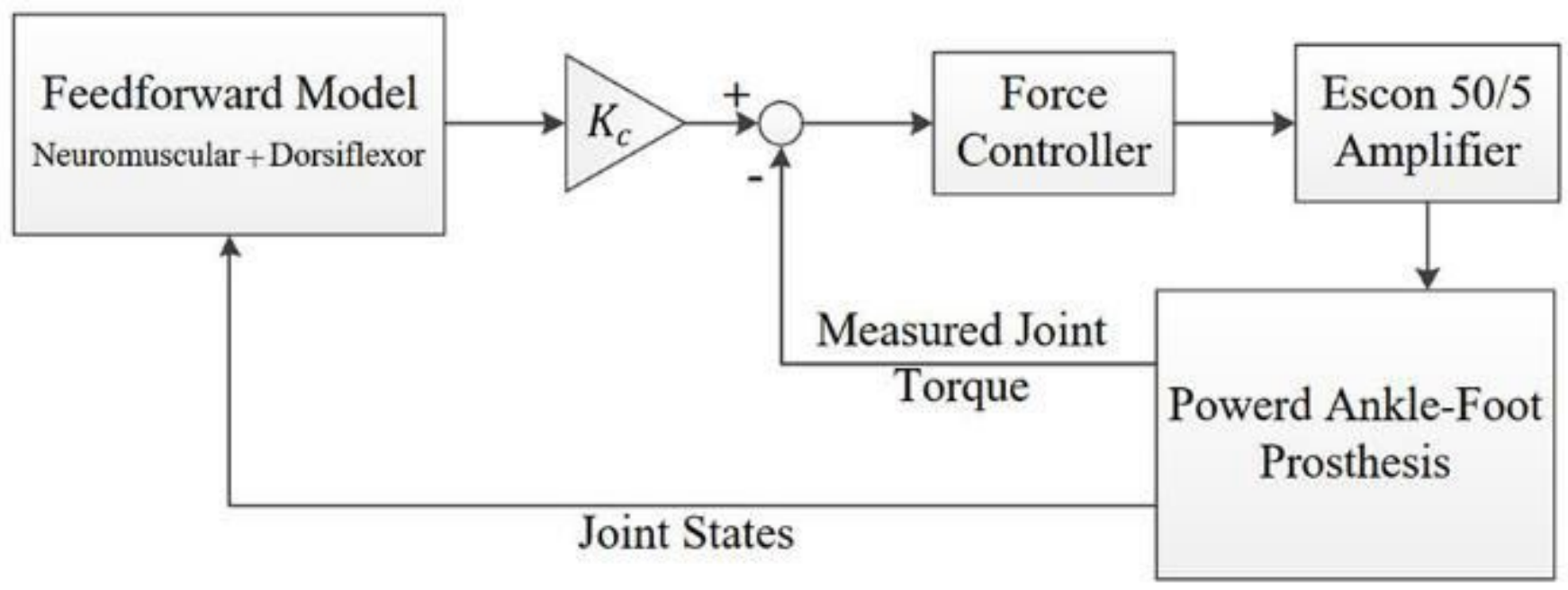

Figure 6

The control system of the powered ankle-foot prosthesis. 


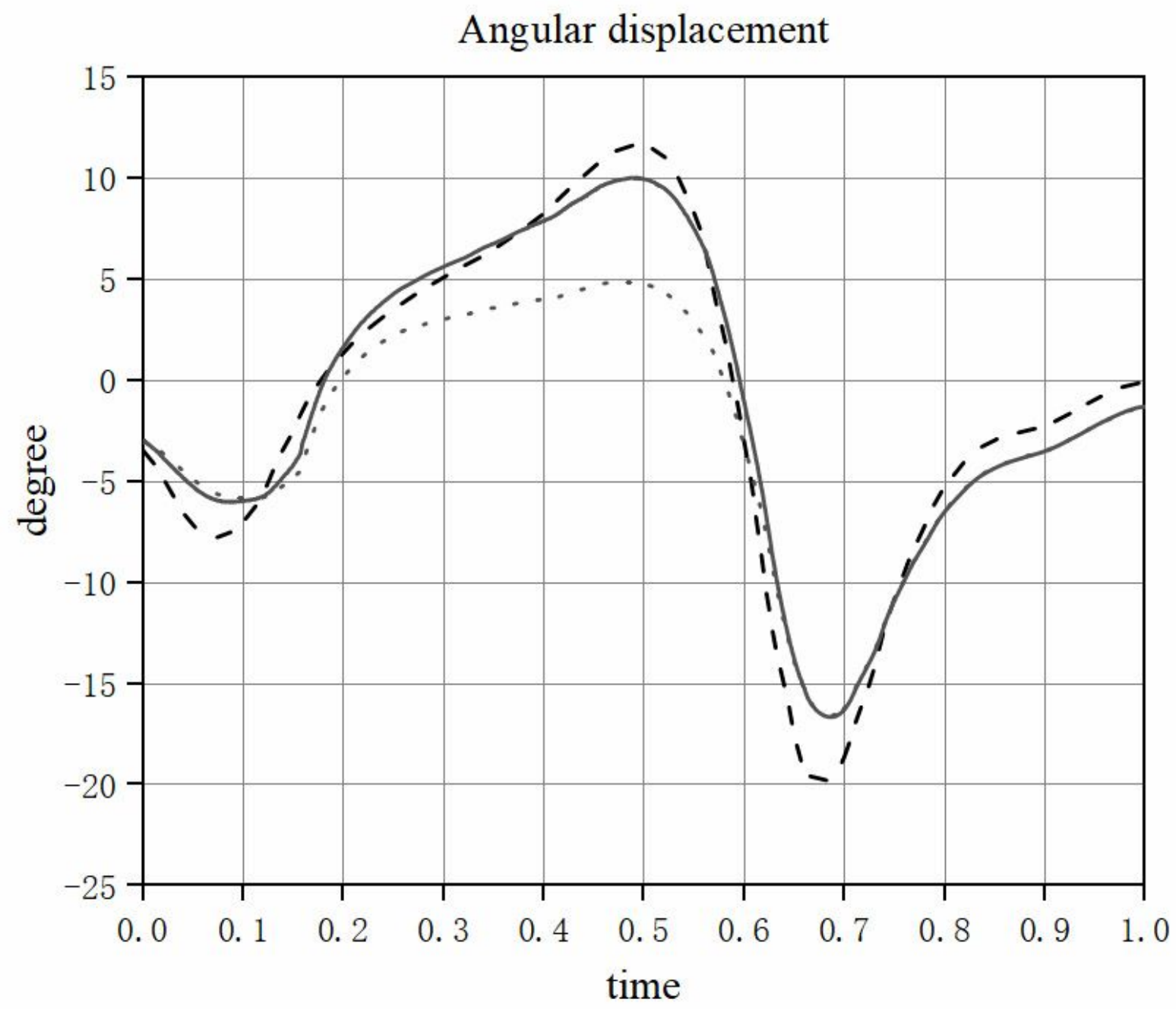

Figure 7

Ankle angle with matched groups of a pre-sampled biological ankle angle profile and the angular displacement of actuator. 


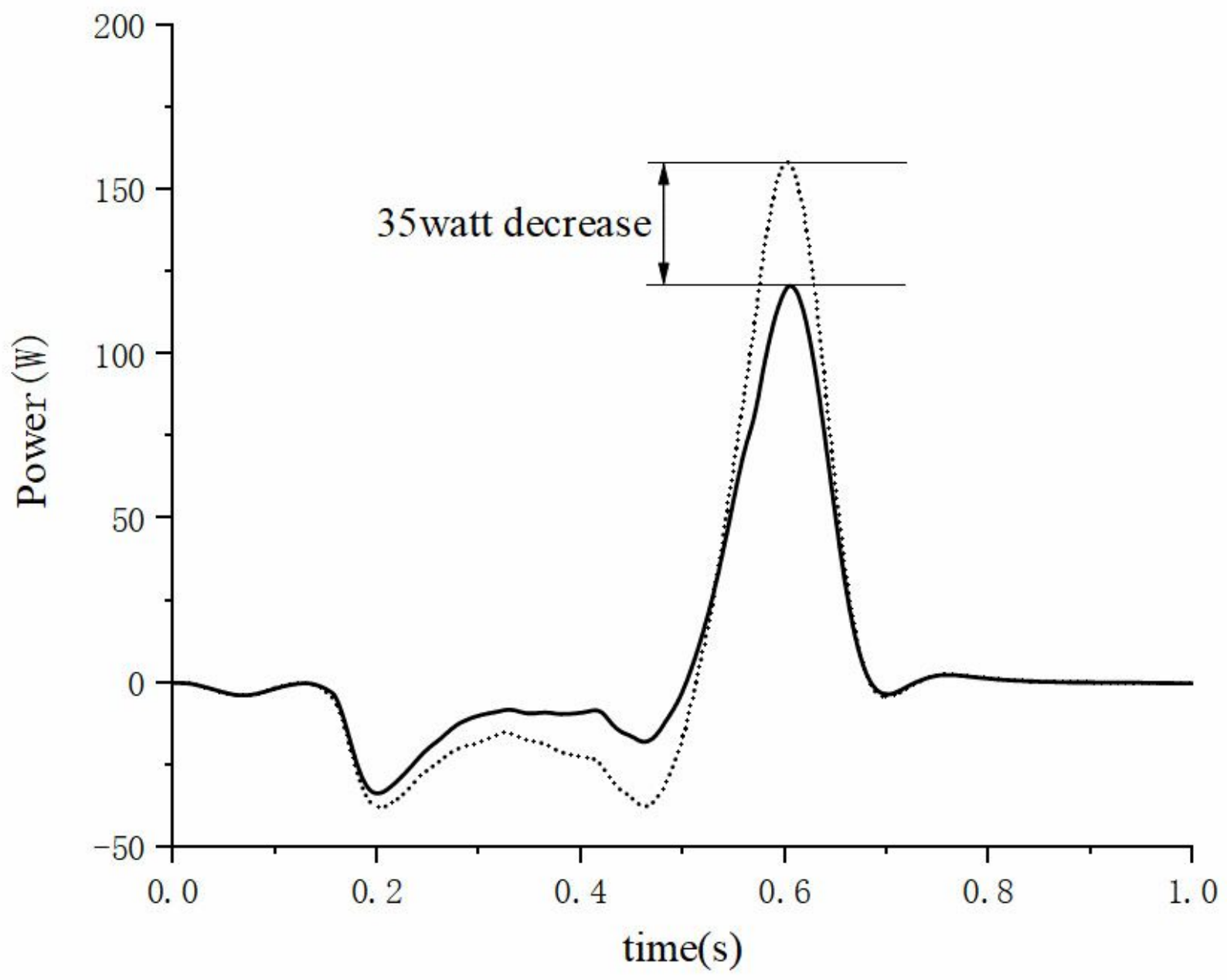

Figure 8

Ankle power output comparison. 